\title{
High expression of monocarboxylate transporter 4 (MCT 4), but not MCT 1, predicts poor prognosis in patients with non-small cell lung cancer
}

\author{
Ying-Hui Tong ${ }^{1}$, Xiao-Ping $\mathrm{Hu}^{2}$, Xue-Ping Xiang ${ }^{3 \#}$, Luo Fang ${ }^{1 \#}$ \\ ${ }^{1}$ Department of Pharmacy, The Cancer Hospital of the University of Chinese Academy of Sciences (Zhejiang Cancer Hospital), Institute of Basic \\ Medicine and Cancer (IBMC), Chinese Academy of Sciences, Hangzhou, China; ${ }^{2}$ Department of Pharmacy, Zhejiang Provincial People's Hospital, \\ Hangzhou, China; ${ }^{3}$ Department of Pathology, The Second Affiliated Hospital, School of Medicine, Zhejiang University, Hangzhou, China \\ Contributions: (I) Conception and design: L Fang, XP Xiang; (II) Administrative support: YH Tong, XP Xiang; (III) Provision of study materials \\ or patients: YH Tong, XP Xiang; (IV) Collection and assembly of data: YH Tong; (V) Data analysis and interpretation: YH Tong, XP Hu ;(VI) \\ Manuscript writing: All authors; (VII) Final approval of manuscript: All authors. \\ \#The authors contributed equally to this work. \\ Correspondence to: Xueping Xiang. Department of Pathology, the Second Affiliated Hospital, School of Medicine, Zhejiang University, Hangzhou \\ 310009, China. Email: xiangxueping@zju.edu.cn; Luo Fang. Cancer Hospital of University of Chinese Academy of Sciences (Zhejiang Cancer \\ Hospital).,1\# East Banshan Road, Hangzhou 310022, China. Email: Fangluo@zjcc.org.cn.
}

Background: The monocarboxylate transporter (MCT) family especially MCT1 and MCT4 have been recognized to play an important role in lactate transport, a key glycolytic product. The expression of MCT1 and MCT4 expression was previously found to be related to poor outcome in various cancer types. In this study, we investigated the expression status of MCT1 and MCT4 and their relationship with prognosis in non-small cell lung cancer (NSCLC).

Methods: Expression of MCT4 and MCT1 in NSCLC tumor and adjacent lung tissues were detected by immunohistochemistry. Kaplan-Meier plots were used to evaluate two proteins' prognostic role, and the logrank test obtained the $\mathrm{P}$ value. For multivariate analysis, the Cox proportional-hazards regression method was performed.

Results: High MCT4 and MCT1 expression was observed in cancer cells, with a rate of $45 \%$ for MCT4 versus $15 \%$ for MCT1 among all NSCLC patients. High expression of MCT4, and not MCT1, was associated with worse overall survival (OS) [hazard ratio $(\mathrm{HR})=1.96(1.06-3.75), \mathrm{P}=0.032$ ] and progressionfree survival (PFS) $[\mathrm{HR}=1.72$ (1.05-2.93), $\mathrm{P}=0.032]$ in NSCLC patients. In our multivariate analysis, advanced cancer stage and high MCT4 level were identified as independent predictive indicators for both PFS [HR(MCT4) =1.888 (1.114-3.199), P=0.018 and OS [HR (MCT4) =2.421 (1.271-4.610), P=0.007]. Subgroup and interaction analyses were also performed in different clinical characteristic groups and no significant differences were observed.

Conclusions: High MCT4 expression is a predictive marker for worse outcome in NSCLC patients.

Keywords: Monocarboxylate transporter 1 (MCT1); monocarboxylate transporter 4 (MCT4); prognosis; nonsmall cell lung cancer (NSCLC)

Submitted Oct 21, 2020. Accepted for publication Jan 27, 2021.

doi: $10.21037 /$ tcr-20-3117

View this article at: http://dx.doi.org/10.21037/tcr-20-3117 


\section{Introduction}

Lung cancer is the most common malignancy worldwide, with high rates of incidence (2.1 million new cases in 2018) and mortality (1.8 million deaths in 2018) (1). Non-small cell lung cancer (NSCLC) accounts for about $83 \%$ of lung cancer cases (2). Although significant progress in the fields of immunotherapy $(3,4)$ and tyrosine kinase inhibitors $(5)$ in the recent decades has prolonged survival time to some extent, the prognosis of advanced and metastatic NSCLC remains unsatisfactory (1). Therefore, searching for new potential treatment targets is worthwhile.

Cancer cells prefer aerobic glycolysis to obtain energy to support their rapid growth, even in the presence of abundant oxygen, a phenomenon known as the 'Warburg effect' (6). As a consequence, a large volume of lactate is produced. For cancer cells to sustain continuous glycolysis, exporting redundant lactate and maintaining intracellular $\mathrm{pH}$ stability is critical. The monocarboxylate transporter (MCT) family, especially MCT1 and MCT4, have been recognized as important lactate transporters. Also, the overexpression of MCT1 and MCT4 has been observed in various cancers (7-9) including esophageal squamous cell carcinoma (10), glioblastoma (11) and breast cancer $(12,13)$, osteosarcoma (14), hepatocellular carcinoma $(15,16)$, oral squamous cell carcinoma (17), prostate cancer (18), and lung adenocarcinoma (19), and has also been associated with survival outcomes. In urothelial carcinoma, increased MCT4 levels, either ribonucleic acid (RNA) or protein, is a predictor of worse overall survival (OS). Silencing of MCT4 has been shown to promote the intracellular accumulation of lactate and tumor growth reduction (20). Besides the expression of MCT4 in cancer cells, MCT4 expression in cancer-associated fibroblasts was also associated with worse prognosis in patient with pancreatic ductal adenocarcinoma (21).

Although both MCT1 and MCT4 have been reported to play an important role in cancer progression, their function and effect on prognosis may be different to some extent. For example, elevated MCT1 levels were indicative factor for worse survival in breast cancer (22). However, in triplenegative breast cancer, MCT4, and not MCT1, had a significant deleterious impact on survival (23). It is suggested that in context of different tumor types, the role of MCT1 and MCT4 may be varying. Besides, although both MCT1 and MCT4 act as lactate and ketone bodies transporters, MCT1 has a much higher affinity for both lactate and pyruvate than MCT4 (24). Also, MCT1 has a bi-directional transport function for lactate shuttle, while MCT4 mainly focuses on lactate export. Thus, a comprehensive evaluation of MCT1 and MCT4 is meaningful. In this study, we investigated both the expression of both MCT1 and MCT4 and examined their association with survival. Our results suggested that MCT4, and not MCT1, is a predictive marker of worse survival outcome in patients with NSCLC. We present the following article in accordance with the REMARK reporting checklist (available at: http://dx.doi. org/10.21037/tcr-20-3117).

\section{Methods}

\section{Patients and samples}

In this retrospective study, primary NSCLC patients who signed informed consent and had well-stored surgical specimens in the biological sample bank of Zhejiang cancer hospital were reviewed. Surgical samples between July 2011 and October 2012 were chosen randomly to ensure surgical homogeneity and sufficient follow-up time to obtain the prognosis data. Tumor tissues and adjacent lung tissues were collected from the tumor tissue bank. TNM stages were evaluated according to the 7 th edition of the international staging system (25). The follow-up work was carried out by reviewing medical records and telephone interviews (up to May 20th, 2019). Progression-free survival (PFS) and OS time were calculated from surgery date to disease progression or death from any cause, respectively. The study was conducted in accordance with the Declaration of Helsinki (as revised in 2013). The study was approved by The Ethics Committee of Zhejiang Cancer Hospital (IRB-2019-41) and individual consent for this retrospective analysis were waived.

\section{Immunobistochemical (IHC) staining and evaluation}

IHC staining was performed to evaluated the expression of MCT1 and MCT4, in both tumors and paired adjacent lung tissues in each case. In brief, the formalin-fixed paraffinembedded tissues were cut into a series of $5 \mu \mathrm{m}$-thick sections. The sections were de-paraffinized, rehydrated and underwent antigen retrieval using Dako EnVisionTM FLEX Target Retrieval Solution (pH 9.0) at $95{ }^{\circ} \mathrm{C}$ for 20 minutes. Anti-SLC16A3 (MCT4) antibody (Rabbit polyclonal antibody, HPA021451, Sigma-Aldrich), and anti-MCT1 antibody (Rabbit polyclonal antibody, ab238825, Abcam) were used as primary antibodies, and were diluted at a ratio 
Table 1 Baseline characteristics of patients

\begin{tabular}{lc}
\hline Characteristics & Values \\
\hline Total, $\mathrm{n}$ & 100 \\
Age, median years (range) & $59[40-79]$ \\
Gender, $\mathrm{n}(\%)$ & \\
Male & $74[74]$ \\
Female & $26[26]$ \\
Pathological types, $\mathrm{n}(\%)$ & \\
Adenocarcinoma & $54[54]$ \\
Squamous & $43[43]$ \\
Others ${ }^{\dagger}$ & $3[3]$ \\
Tumor differentiation, $\mathrm{n}(\%)$ & \\
Poor & $52[52]$ \\
Moderate & $48[48]$ \\
Stage, $\mathrm{n}(\%)$ & \\
I-II & \\
III-IV & $71[71]$ \\
Smoking history, $\mathrm{n}(\%)$ & $29[29]$ \\
Never & \\
Smoker & \\
\hline & \\
\hline
\end{tabular}

${ }^{\dagger}$, others including adenosquamous carcinoma, large cell carcinoma, sarcoma, etc.

of 1:100. After 2 hours of primary antibody incubation at room temperature, incubation with a secondary antibody (PV-9000, Zhongshan Jinqiao Biotechnology Co. Ltd., Beijing, China) was performed at room temperature for 30 minutes. Finally, 3,3'-diaminobenzamine and hematoxylin were used for coloration of the immune complex and nucleus, respectively. The expression of MCT4 and MCT1 was assessed by multiplying the staining intensity score and the percentage score as described in our previous study (26). A final score $\geq 6$ was defined as high expression.

\section{Statistical analyses}

SPSS software for Windows (Version 22.0, SPSS, Inc., Chicago, IL, USA) and GraphPad Prism (Version 5.0, GraphPad Software, San Diego, CA, USA) were used for statistical analyses and graphic processing. Kaplan-Meier analysis with log-rank test was performed for survival analysis. The Cox proportional-hazards regression method was used for multivariate analysis. For baseline evaluation of different clinicopathological groups, the Chi-squared $\left(\chi^{2}\right)$ and Fisher's exact tests were performed. For all tests, $\mathrm{P}$ values were two-sided and $\mathrm{P}<0.05$ was considered statistically significant.

\section{Results}

\section{Patients}

The baseline characteristics of 100 patients are shown in Table 1. The median age of the patients was is 59 years (range, 40-79). Most of the patients were male (74\%), at an early stage (stages I \& II, 71\%), or had a history of with smoking (67\%). During the 8-year follow-up, 40 patients died, and 61 patients experienced disease progression, with a median OS of 58.3 [95\% confidence interval (CI): 49.6-61.5] months and a PFS of 23.8 (95\% CI: 29.6-42.8) months, respectively.

\section{Expression of MCT1 and MCT4}

MCT1 and MCT4 staining was mainly localized in the plasma membrane (Figure 1). The definition of positive/ high expression of MCT1 and MCT4 have been described above. Different expression level of MCT1 and MCT4 were observed in NSCLC cells (Figure 1A,B). A high expression of both MCT1 (Figure 1C) and MCT4 (Figure 1D) were detected in cancer cells. The expression of MCT1 and MCT4 was negative/low in adjacent nontumor lung tissues. High MCT4 expression was found more frequently in cancer cells than MCT1 expression, with a positive rate of $45 \%$ for MCT 4 versus $15 \%$ for MCT1 among all samples. The correlation between MCT1 and MCT4 expression is shown in Table S1. The expression status of MCT1 and MCT4 in patients with different clinicopathological characteristics is depicted in Table 2. There was no significant bias of MCT4 expression in different clinicopathological characteristics subgroups. In contrast, in our study, $100 \%$ negative expression of MCT1 was observed in adenocarcinoma and in female cases.

\section{High expression of MCT4 predicts worse OS and PFS of NSCLC patients}

We then evaluated the association of MCT4 expression with OS and PFS using Kaplan Meier analysis. Our results revealed that high MCT4 expression indicated both shorter 

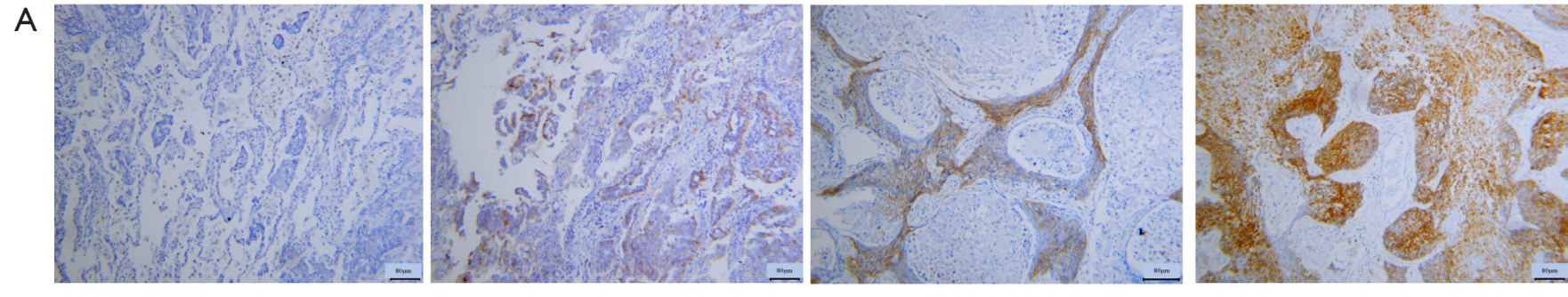

B
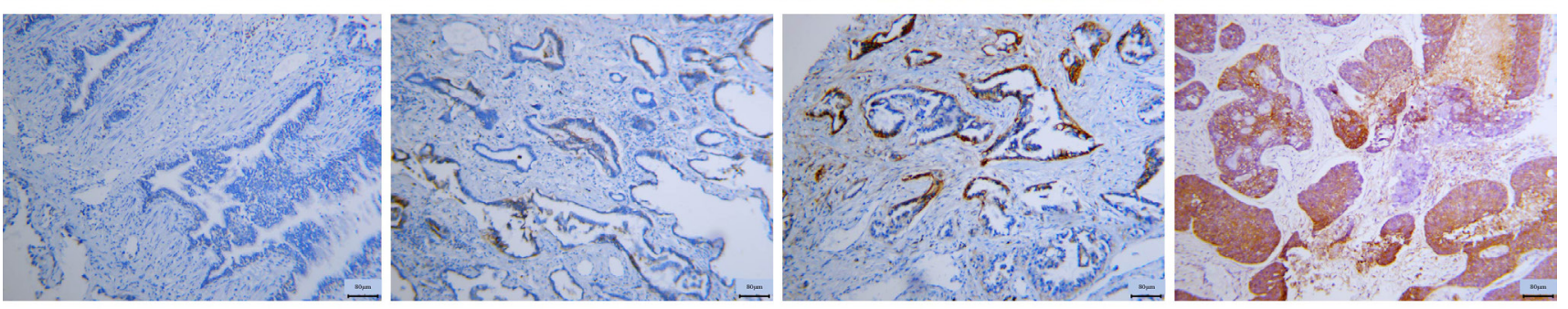

C
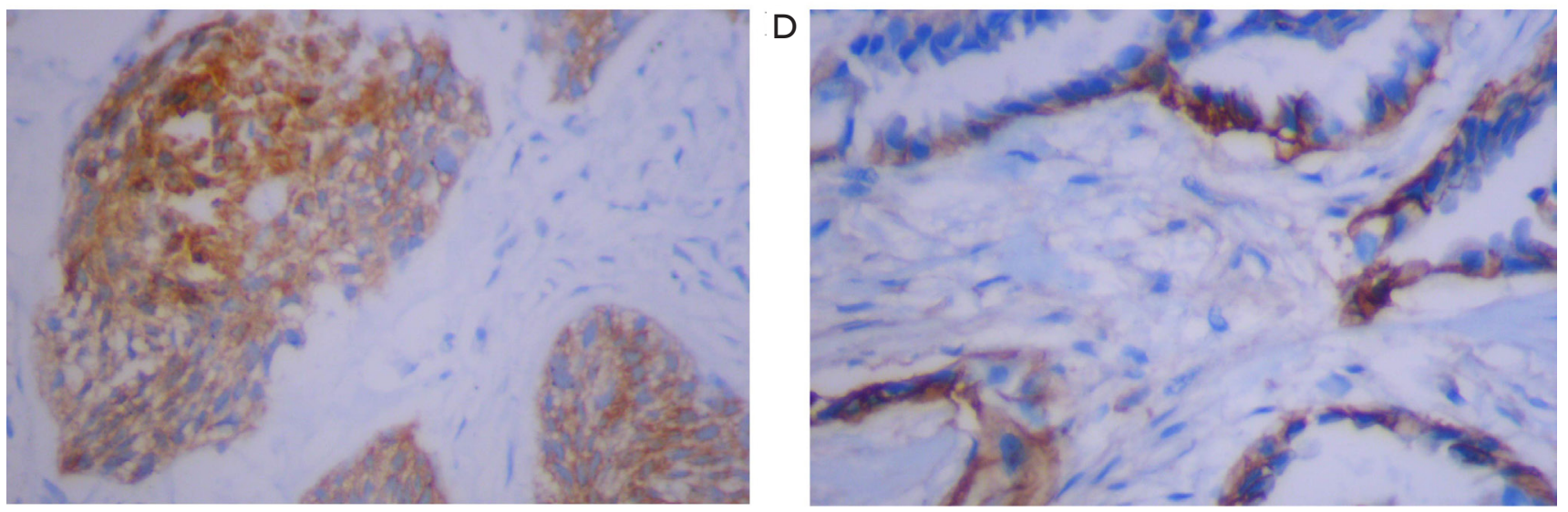

Figure 1 MCT1/4 expression in tumor cells and adjacent lung tissues. (A) The different expression levels of MCT1 in NSCLC cells (negative, low, moderate, high, 4×). (B) Different expression levels of MCT4 in NSCLC cells (negative, low, moderate, high, 4x). (C) High expression of MCT1 in NSCLC tumor cells (40x). (D) High expression of MCT1 in NSCLC tumor cells (40x). MCT1, monocarboxylate transporter 1; MCT4, monocarboxylate transporter 4; NSCLC, non-small cell lung cancer.

OS $[\mathrm{HR}=1.96(1.06-3.75), \mathrm{P}=0.032]$ and $\mathrm{PFS}[\mathrm{HR}=1.72$ (1.05-2.93), $\mathrm{P}=0.032]$ (Figure $2 A, B$ ). In contrast, MCT1 expression was not associated with either OS $[\mathrm{HR}=1.25$ $(0.55-2.91), \mathrm{P}=0.575]$ or PFS $[\mathrm{HR}=0.68(0.37-1.36)$, $\mathrm{P}=0.302]$ (Figure 2C,D).

To further elucidate the independent role of MCT4 in prognostic prediction, MCT1, MCT4 and other clinical characteristics were analyzed using Cox proportional hazards analysis, and the results are shown in Table 3. Advanced cancer stage and MCT4 were identified as predictive indicators for both PFS [HR (MCT4) $=1.888(1.114-3.199)$, $\mathrm{P}=0.018]$ and $\mathrm{OS}[\mathrm{HR}(\mathrm{MCT} 4)=2.421(1.271-4.610)$, $\mathrm{P}=0.007]$, while pathological types and differentiation status of tumor cells were the rest prognostic factor for PFS. In our multivariate analysis, high expression of MCT4 and advanced stage were two independent prognostic factors for both PFS and OS. In addition, the poor differentiation status of cancer cells was another independent prognostic factor for PFS.

\section{Subgroup analyses}

To further clarify the advantage population for MCT4 to predict outcomes, a series of subgroup and interaction analyses were performed in different clinical characteristic groups (Figure 3). MCT4 seemed to be more predictive of OS in elderly patients ( $\geq 60$ years), patients with squamous cell carcinoma, tumors with poor differentiation, advanced stages, smokers, and PFS in males, patients with squamous cell carcinoma, tumor with poor differentiation, 
Table 2 Expression of MCT1 and MCT4 in different characteristics' groups

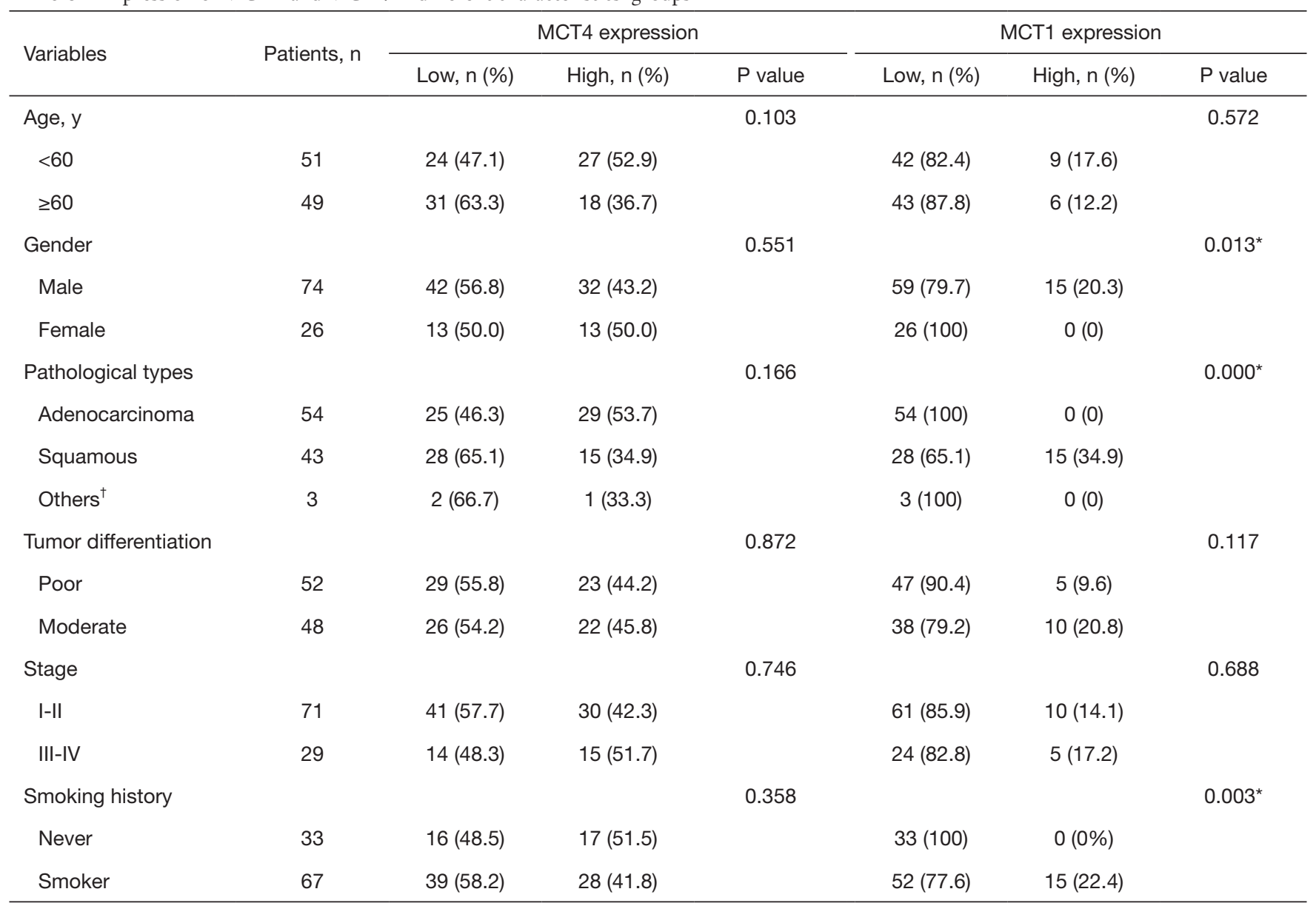

${ }^{\dagger}$, others including adenosquamous carcinoma, large cell carcinoma, sarcoma, etc. Cross-table analysis with Chi-squared $\left(\chi^{2}\right)$ tests were performed. ${ }^{*} \mathrm{P}<0.05$.

and smokers for PFS. However, there was no significant difference in $\mathrm{HR}$ in the interaction analyses (all $\mathrm{P}$ values were $>0.05$ ).

\section{Discussion}

Glycolysis and reprogramming energy metabolism was proposed as a new hallmark of cancer (27). During the past time, lactate was considered to be a metabolic waste in glycolysis. However, increasing evidences has shown that lactate exerts multiple activities in tumor cells' biological function (28). Lactate can be utilized as a source of energy for oxidative cancer cells, function as a pro-angiogenic agent, and also play crucial roles in stimulating amino acid metabolism, inhibiting histone deacetylases and inducing immune tolerance, et al. (29). Thus, how to properly deal with lactate is of great significance in glycolytic cancer cells. MCT family members plays an important role in lactate transport, especially MCT1 and MCT4. These two proteins were found widely expressed in various malignancies and play multiple roles in cancer, not merely limited to monocarboxylate transport, but also in metabolic exchanges, metabolic signaling, and cancer metastasis.

In this study, we reported that the expression of MCT4, but not MCT1, was associated with worse OS and PFS in NSCLC patients. We also tested whether the combination of MCT1/4 IHC staining ("any" or "both") is a better prognostic factor. Since there were only five cases are both MCT1/4 high expression (data are shown in Table S1), we examined the predictive role of MCT1/4 (+), which was defined as MCT1 or MCT4 positive. In our study, MCT1/4 (+) predicted worse OS for NSCLC patients 
A

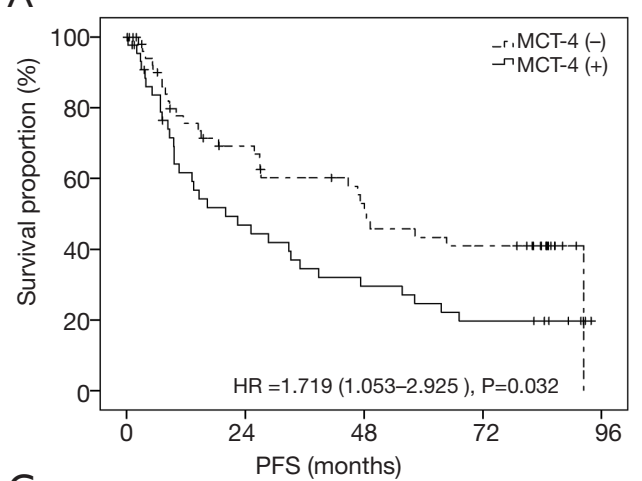

C

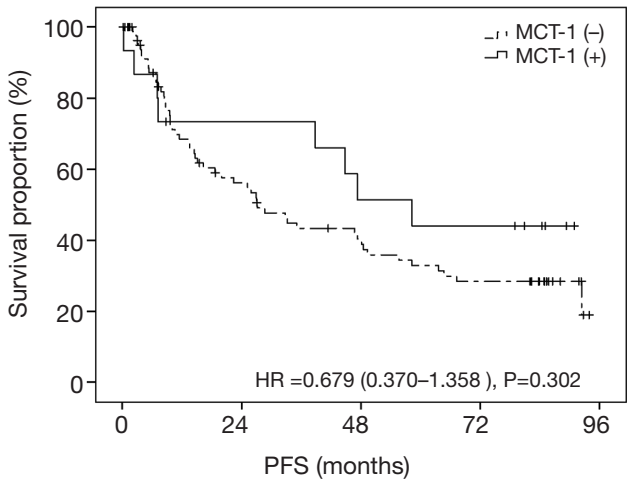

B

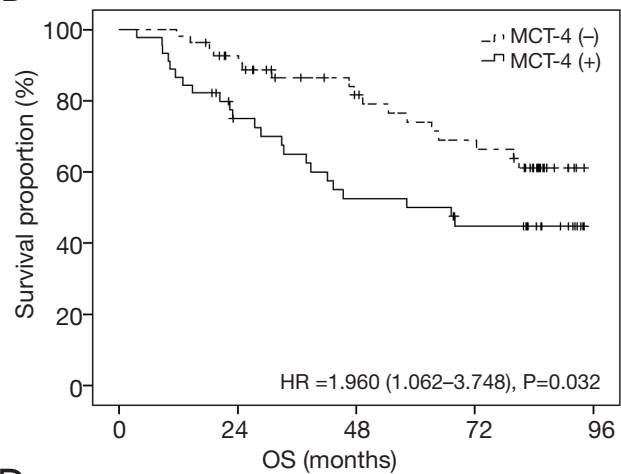

$\mathrm{D}$

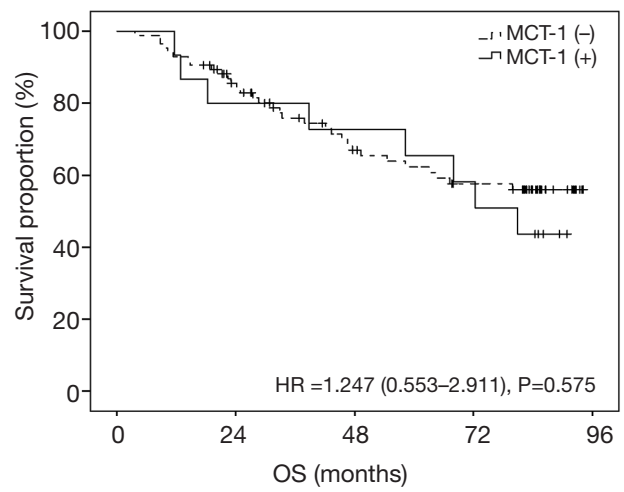

Figure 2 High expression of monocarboxylate transporter 4 MCT 4, but not MCT 1, predicts worse prognosis in patients with NSCLC. (A) High MCT4 expression was associated with worse PFS. (B) High MCT4 expression was associated with worse OS, P=0.032. (C) MCT1 expression was not significantly associated with PFS outcome. (D) MCT1 expression was not significantly associated with OS outcome. MCT1, monocarboxylate transporter 1; MCT4, monocarboxylate transporter 4; NSCLC, non-small cell lung cancer; PFS, progression-free survival; OS, overall survival.

[HR =2.142 (1.101-3.807), $\mathrm{P}=0.024)]$, however, it failed to be a prognostic factor for PFS [HR $=1.501(0.901-2.462)$, $\mathrm{p}=0.121$ )] (Figure S1). Thus, our results demonstrated that MCT4 is a better prognostic factor for NSCLC patients. As mentioned above, aerobic glycolysis is an essential step in the metabolic reprogramming of cancer cells. In this process, MCT1 and MCT4 play important roles in lactate transport and maintaining intracellular $\mathrm{pH}$. Although MCT1 has a much higher affinity for lactate transport than MCT4 (24), in our study, we found high MCT4 expression, instead of MCT1 expression, was an independent indicator for worse prognosis. In addition, the expression of MCT4 was more frequently observed in NSCLC tumor cells.

There are several potential mechanisms to explain why tumoral MCT4 levels, but not MCT1, are more relevant to a worse prognosis. Firstly, compared with MCT4, MCT1 has markedly higher affinity, not only for lactate transport, but also for other substrates such as pyruvate, et al. (24).
On the contrary, MCT4 exhibits high specificity for lactate transport, with lower affinity and high $\mathrm{K}_{\mathrm{m}}$ and $\mathrm{K}_{\mathrm{i}}$ values for other substrates $(24,30)$. The excessive loss of pyruvate from the cell may reduce the renewal of nicotinamide adenine dinucleotide (NAD+) during lactate production, which is necessary for maintaining a high glycolytic flux. Treatment with an MCT1 inhibitor in vitro were reported to slightly promoted cell growth in most glycolytic cells (23). Secondly, MCT1 also acts as a bi-directional transport through lactate shuttle in specific cells and tumor-stroma interplay, while MCT4 mainly focus on the export of lactate (31). Exporting lactate to the extracellular matrix were proved crucial for oxygenation of cancer cells, which used lactate as a sources of energy (29). Meanwhile MCT4 expression markedly increased the $\mathrm{pH}$ value gradient between the intracellular and extracellular environments, paralleled by increased tumor growth and diminished necrotic regions (32). Thirdly, the presence of hypoxia response elements (HREs) 
Table 3 Cox proportional hazards analyses for progression-free survival and overall survival

\begin{tabular}{|c|c|c|c|c|}
\hline Variables & \multicolumn{2}{|c|}{ PFS } & \multicolumn{2}{|c|}{ OS } \\
\hline \multicolumn{5}{|l|}{ Univariate } \\
\hline Age, y (<60 vs. $\geq 60)$ & $0.809(0.488-1.341)$ & 0.411 & $0.948(0.510-1.764)$ & 0.867 \\
\hline Gender (male vs. female) & $1.417(0.816-2.462)$ & 0.216 & $0.896(0.438-1.833)$ & 0.763 \\
\hline Tumor differentiation (poor vs. moderate) & $0.513(0.306-0.861)$ & $0.012^{*}$ & $0.638(0.341-1.194)$ & 0.160 \\
\hline TNM Stage (I-II vs. III-IV) & $2.792(1.654-4.711)$ & $0.000^{*}$ & $3.504(1.880-6.530)$ & $0.000^{*}$ \\
\hline Smoking history (never vs. smoker) & $0.756(0.446-1.279)$ & 0.297 & $1.367(0.683-2.738)$ & 0.377 \\
\hline $\begin{array}{l}\text { Pathological types (adenocarcinoma vs. } \\
\text { squamous vs. others) }\end{array}$ & $0.788(0.472-1.317)$ & 0.363 & 1.609 (0.899-2.877 & 0.109 \\
\hline Tumor differentiation (poor vs. moderate) & $0.555(0.322-0.957)$ & $0.034^{\star}$ & $0.752(0.394-1.437)$ & 0.389 \\
\hline TNM Stage (I-II vs. III-IV) & $2.492(1.442-4.307)$ & $0.001^{*}$ & $3.725(1.957-7088)$ & $0.000^{*}$ \\
\hline MCT-4 expression (low vs. high) & $1.888(1.114-3.199)$ & $0.018^{\star}$ & $2.421(1.271-4.610)$ & $0.007^{\star}$ \\
\hline
\end{tabular}
${ }^{*} \mathrm{P}<0.05$.

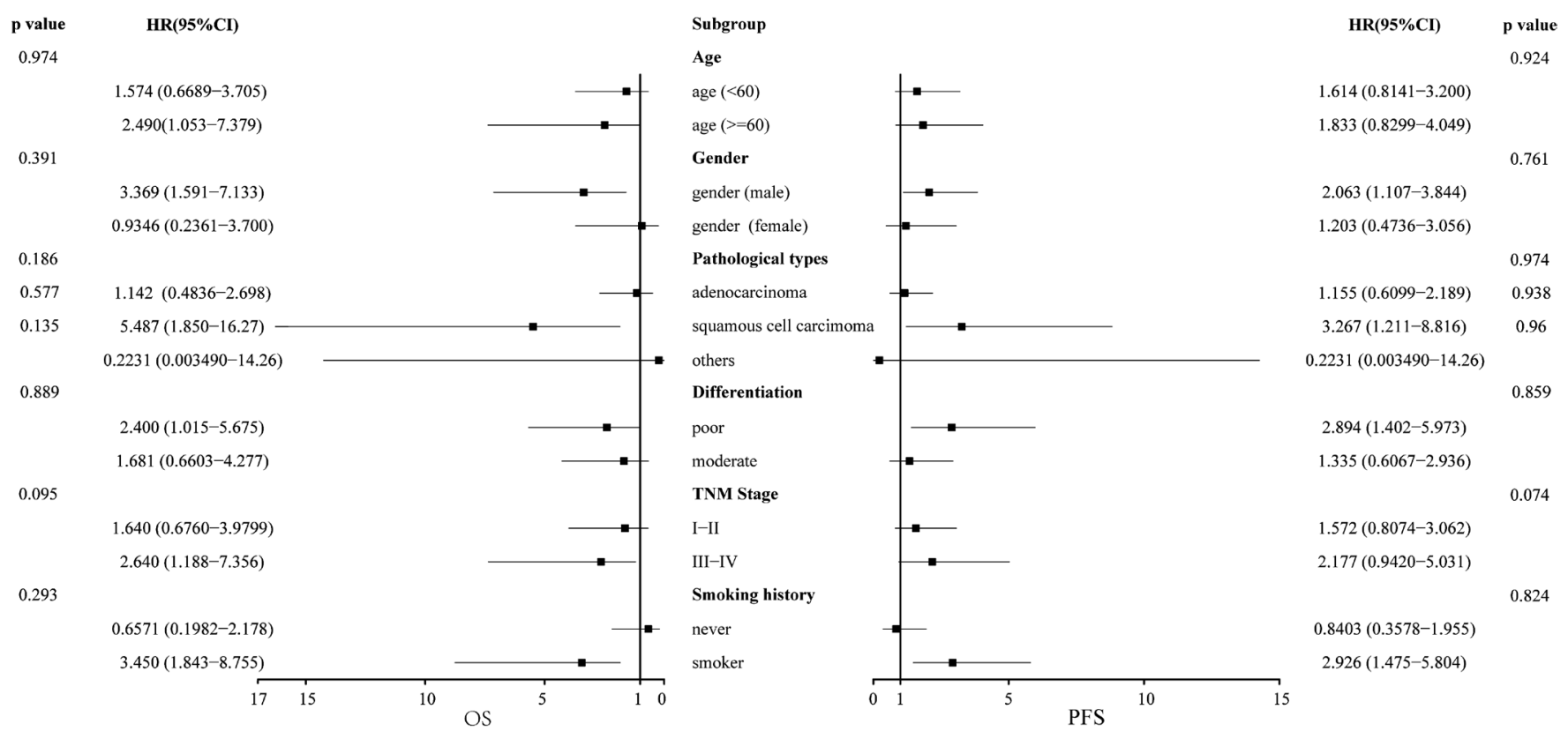

Figure 3 Subgroup and interaction analyses for MCT4 in predicting PFS and OS. No significant difference was observed in the interaction analyses (all P values are >0.05). MCT4, monocarboxylate transporter 4; PFS, progression-free survival; OS, overall survival. 
in the promoter region of MCT4 makes it a direct hypoxia induced factor (HIF)-1-target gene $(33,34)$. Thus, MCT4 expression in the tumor may reflect HIF-1 activation, which promotes epithelial to mesenchymal transition (EMT), angiogenesis, and metastasis of tumor cells. Finally, MCT4 expression was previously found to be abundant in the neonatal heart of rat, but was absent in the adult heart, where MCT1 expression is high (35), suggesting that MCT4 expression is more likely to occur in glycolytic cells (35).

Ruan et al. reported that high MCT4 expression is predictive in patients with lung adenocarcinoma. However, their study did not mention whether MCT4 is a prognostic factor in other types of NSCLC, such as squamous-cell carcinoma. Our study's subgroup analyses revealed a trend that MCT4 seemed to be more predictive in patients with squamous cell carcinoma (Figure 3). However, our study found no significant difference in the interaction analyses (all $\mathrm{P}$ values are $>0.05)$.

Further studies may be required to explore and demonstrate the potential mechanisms. Based on our findings and those of numerous studies focus on the functions of MCT4 in cancer, we believe that MCT4 has great potential to be an anticancer target for patient treatment. Although MCT1 inhibitors such as AZD3965 has been entering clinical trials for several types of advanced cancer (NCT01791595), selective MCT4 inhibitors are still in the discovery phase. We hope that development of MCT4 inhibitors in the future will offer a new approach to cancer therapy.

\section{Conclusions}

High MCT4 expression is an independent predictive marker for worse outcomes in NSCLC.

\section{Acknowledgments}

Funding: This work was supported by the National Natural Science Foundation of China (81803549 to XuePing Xiang), the Natural Science Foundation of Zhejiang Province (LQ18H310002 to Xue-Ping Xiang), and Projects of Medical and Health Technology Program in Zhejiang Province (2018253186 to Ying-Hui Tong, 2015KYB061).

\section{Footnote}

Reporting Checklist: The authors have completed the
REMARK reporting checklist. Available at http://dx.doi. org/10.21037/tcr-20-3117

Data Sharing Statement: Available at http://dx.doi. org/10.21037/tcr-20-3117

Conflicts of Interest: All authors have completed the ICMJE uniform disclosure form (available at http://dx.doi. org/10.21037/tcr-20-3117). The authors have no conflicts of interest to declare.

Ethical Statement: The authors are accountable for all aspects of the work in ensuring that questions related to the accuracy or integrity of any part of the work are appropriately investigated and resolved. The study was conducted in accordance with the Declaration of Helsinki (as revised in 2013). The study was approved by The Ethics Committee of Zhejiang Cancer Hospital (IRB-2019-41) and individual consent for this retrospective analysis were waived.

Open Access Statement: This is an Open Access article distributed in accordance with the Creative Commons Attribution-NonCommercial-NoDerivs 4.0 International License (CC BY-NC-ND 4.0), which permits the noncommercial replication and distribution of the article with the strict proviso that no changes or edits are made and the original work is properly cited (including links to both the formal publication through the relevant DOI and the license). See: https://creativecommons.org/licenses/by-nc-nd/4.0/.

\section{References}

1. Edited by Wild CP WE, Stewart BW. World Cancer Report: Cancer Research for Cancer Prevention 2020. 2020.

2. Miller KD, Nogueira L, Mariotto AB, et al. Cancer treatment and survivorship statistics, 2019. CA Cancer J Clin 2019;69:363-85.

3. Wang L, Ma Q, Yao R, et al. Current status and development of anti-PD-1/PD-L1 immunotherapy for lung cancer. Int Immunopharmacol 2020;79:106088.

4. Andrews LP, Yano H, Vignali DAA. Inhibitory receptors and ligands beyond PD-1, PD-L1 and CTLA-4: breakthroughs or backups. Nat Immunol 2019;20:1425-34.

5. Buonerba C, Iaccarino S, Dolce P, et al. Predictors of Outcomes in Patients with EGFR-Mutated Non-Small Cell Lung Cancer Receiving EGFR Tyrosine Kinase 
Inhibitors: A Systematic Review and Meta-Analysis.

Cancers (Basel) 2019;11:1259.

6. Liberti MV, Locasale JW. The Warburg Effect: How Does it Benefit Cancer Cells? Trends Biochem Sci 2016;41:211-8.

7. Fang J, Quinones QJ, Holman TL, et al. The H+-linked monocarboxylate transporter (MCT1/SLC16A1): a potential therapeutic target for high-risk neuroblastoma. Mol Pharmacol 2006;70:2108-15.

8. Curry JM, Tuluc M, Whitaker-Menezes D, et al. Cancer metabolism, stemness and tumor recurrence: MCT1 and MCT4 are functional biomarkers of metabolic symbiosis in head and neck cancer. Cell Cycle 2013;12:1371-84.

9. Kim Y, Choi JW, Lee JH, et al. Expression of lactate/ $\mathrm{H}(+)$ symporters MCT1 and MCT4 and their chaperone CD147 predicts tumor progression in clear cell renal cell carcinoma: immunohistochemical and The Cancer Genome Atlas data analyses. Hum Pathol 2015;46:104-12.

10. Chen X, Chen X, Liu F, et al. Monocarboxylate transporter 1 is an independent prognostic factor in esophageal squamous cell carcinoma. Oncol Rep 2019;41:2529-39.

11. Duan K, Liu ZJ, Hu SQ, et al. Lactic acid induces lactate transport and glycolysis/OXPHOS interconversion in glioblastoma. Biochem Biophys Res Commun 2018;503:888-94.

12. Li Z, Wu Q, Sun S, et al. Monocarboxylate transporters in breast cancer and adipose tissue are novel biomarkers and potential therapeutic targets. Biochem Biophys Res Commun 2018;501:962-7.

13. Xiao S, Zhu H, Shi Y, et al. Prognostic and predictive value of monocarboxylate transporter 4 in patients with breast cancer. Oncol Lett 2020;20:2143-52.

14. Liu Y, Sun X, Huo C, et al. Monocarboxylate Transporter 4 (MCT4) Overexpression Is Correlated with Poor Prognosis of Osteosarcoma. Med Sci Monit 2019;25:4278-84.

15. Chen HL, OuYang HY, Le Y, et al. Aberrant MCT4 and GLUT1 expression is correlated with early recurrence and poor prognosis of hepatocellular carcinoma after hepatectomy. Cancer Med 2018;7:5339-50.

16. Gao HJ, Zhao MC, Zhang YJ, et al. Monocarboxylate transporter 4 predicts poor prognosis in hepatocellular carcinoma and is associated with cell proliferation and migration. J Cancer Res Clin Oncol 2015;141:1151-62.

17. Zhu J, Wu YN, Zhang W, et al. Monocarboxylate transporter 4 facilitates cell proliferation and migration and is associated with poor prognosis in oral squamous cell carcinoma patients. PLoS One 2014;9:e87904.
18. Pertega-Gomes N, Vizcaino JR, Miranda-Goncalves V, et al. Monocarboxylate transporter 4 (MCT4) and CD147 overexpression is associated with poor prognosis in prostate cancer. BMC Cancer 2011;11:312.

19. Ruan Y, Zeng F, Cheng Z, et al. High expression of monocarboxylate transporter 4 predicts poor prognosis in patients with lung adenocarcinoma. Oncol Lett 2017;14:5727-34.

20. Todenhofer T, Seiler R, Stewart C, et al. Selective Inhibition of the Lactate Transporter MCT4 Reduces Growth of Invasive Bladder Cancer. Mol Cancer Ther 2018;17:2746-55

21. Sukeda A, Nakamura Y, Nishida Y, et al. Expression of Monocarboxylate Transporter 1 Is Associated With Better Prognosis and Reduced Nodal Metastasis in Pancreatic Ductal Adenocarcinoma. Pancreas 2019;48:1102-10.

22. Hong CS, Graham NA, Gu W, et al. MCT1 Modulates Cancer Cell Pyruvate Export and Growth of Tumors that Co-express MCT1 and MCT4. Cell Rep 2016;14:1590-601.

23. Doyen J, Trastour C, Ettore F, et al. Expression of the hypoxia-inducible monocarboxylate transporter MCT4 is increased in triple negative breast cancer and correlates independently with clinical outcome. Biochem Biophys Res Commun 2014;451:54-61.

24. Halestrap AP. The monocarboxylate transporter family-Structure and functional characterization. IUBMB Life 2012;64:1-9.

25. Sobin L H GMKW. International Union Against Cancer (UICC) TNM Classification of Malignant Tumors. Oxford, UK: Wiley-Blackwell, 2009.

26. Xiang X, Ma X, Fang M, et al. Jumonji domain containing 5 is a potential prognostic indicator in non-small cell lung cancer patients who received platinum-based chemotherapy. 2019;8:2535-42.

27. Hanahan D, Weinberg RA. Hallmarks of cancer: the next generation. Cell 2011;144:646-74.

28. Payen VL, Mina E, Van Hee VF, et al. Monocarboxylate transporters in cancer. Mol Metab 2020;33:48-66.

29. Faubert B, Li KY, Cai L, et al. Lactate Metabolism in Human Lung Tumors. Cell 2017;171:358-71.e9.

30. Contreras-Baeza Y, Sandoval PY, Alarcon R, et al. Monocarboxylate transporter 4 (MCT4) is a high affinity transporter capable of exporting lactate in high-lactate microenvironments. J Biol Chem 2019;294:20135-47.

31. Fiaschi T, Marini A, Giannoni E, et al. Reciprocal metabolic reprogramming through lactate shuttle coordinately influences tumor-stroma interplay. Cancer 
Res 2012;72:5130-40.

32. Chiche J, Le Fur Y, Vilmen C, et al. In vivo $\mathrm{pH}$ in metabolic-defective Ras-transformed fibroblast tumors: key role of the monocarboxylate transporter, MCT4, for inducing an alkaline intracellular $\mathrm{pH}$. Int $\mathrm{J}$ Cancer 2012;130:1511-20.

33. Khan A, Valli E, Lam H, et al. Targeting metabolic activity in high-risk neuroblastoma through Monocarboxylate Transporter 1 (MCT1) inhibition. Oncogene

Cite this article as: Tong YH, Hu XP, Xiang XP, Fang L. High expression of monocarboxylate transporter 4 (MCT 4), but not MCT 1, predicts poor prognosis in patients with non-small cell lung cancer. Transl Cancer Res 2021;10(3):1336-1345. doi: $10.21037 /$ tcr-20-3117
2020;39:3555-70.

34. Ullah MS, Davies AJ, Halestrap AP. The plasma membrane lactate transporter MCT4, but not MCT1, is up-regulated by hypoxia through a HIF-1alpha-dependent mechanism. J Biol Chem 2006;281:9030-7.

35. Hatta H, Tonouchi M, Miskovic D, et al. Tissue-specific and isoform-specific changes in MCT1 and MCT4 in heart and soleus muscle during a 1-yr period. Am J Physiol Endocrinol Metab 2001;281:E749-56. 


\section{Supplementary}

Table S1 The correlation between MCT1 and MCT4 expression status.

\begin{tabular}{lcccc}
\hline & & & MCT4 & Total \\
\hline MCT1 & Low & High & & 0.329 \\
Low & & & 85 \\
High & 45 & 40 & 15 \\
Total & 10 & 5 & 100 \\
\hline
\end{tabular}

The correlation between MCT1 and MCT4 expression was evaluated by Pearson Correlation test. MCT1: monocarboxylate transporter 1; MCT4: monocarboxylate transporter 4.
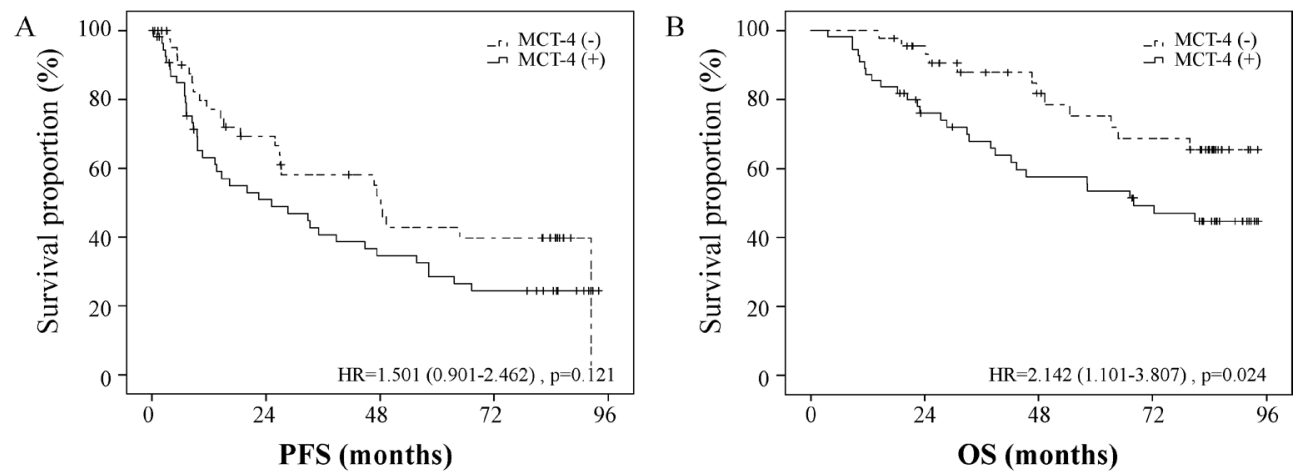

Figure S1 Survival analysis of MCT 1/4 expression, performed by Kaplan-Meier analysis with log-rank test. (A) Survival analysis of PFS. (B) Survival analysis of OS. MCT1/4 (+): High expression of MCT1 or MCT4. MCT1/4 (-): Low expression of both MCT1 and MCT4. MCT1, monocarboxylate transporter 1; MCT4, monocarboxylate transporter 4; PFS, progression-free survival; OS, overall survival. 